\title{
Ausencia Congénita de la Vena Porta Hepática Asociada a Malrotación Intestinal y a Vena Cava Inferior Izquierda. Reporte de Caso
}

\author{
Congenital Absence of The Hepatic Portal Vein Associated to Intestinal \\ Malrotation and Left-Sided Inferior Vena Cava. A Case Report
}

Oscar Inzunza*; Cristian J. García**; Sergio Zúñiga $(\dagger)^{* * *}$; Martín Inzunza*; Daniela Schneeberger*; Guillermo Salgado* \& Emilio Farfán*

INZUNZA, O.; GARCÍA, C. J.; ZÚÑIGA, S.; INZUNZA, M.; SCHNEEBERGER, D.; SALGADO, G. \& FARFÁN, E. Ausencia congénita de la vena porta hepática asociada a malrotacion intestinal y a vena cava inferior izquierda. Reporte de caso. Int. J. Morphol., 34(2):665-670, 2016.

RESUMEN: La ausencia congénita de la vena porta hepática es una malformación en extremo rara, fue descrita por primera vez en 1793 por John Abernethy y a la fecha se han reportado sólo 101 casos. Afecta con mayor frecuencia a mujeres y determina que el drenaje venoso intestinal sea derivado hacia el territorio de las venas sistémicas. Es también conocida como derivación porto-sistémica extra hepática congénita (CEPS), por su sigla en inglés, y se asocia a otras alteraciones congénitas, incluyendo anomalías cardíacas, de las vías biliares, enfermedades metabólicas y retardo mental. En este trabajo presentamos el hallazgo de esta malformación en el cadáver de un niño de dos años de edad, donde la vena porta seguía un trayecto anómalo y se unía a la vena renal derecha. El confluente venoso "mesentérico-esplénico-renal" así formado presentaba un trayecto descendente, recibía a la vena gonadal derecha, y desembocando en la confluencia de las venas ilíacas comunes. Esto se asociaba a mal rotación intestinal, arteria hepática aberrante y a vena cava inferior izquierda, situación descrita sólo una vez en la literatura. El hallazgo de estas anomalías anatómicas en los cadáveres disecados con fines docentes en nuestro Departamento de Anatomía, tiene un valor formativo indiscutible para nuestros alumnos de pre y postgrado, quienes pueden valorar las implicancias de este conocimiento anatómico en la clínica diaria.

PALABRAS CLAVES: Ausencia de vena porta hepática; Anastomosis porto-cava; Mal rotación intestinal; Vena cava inferior izquierda.

\section{INTRODUCCIÓN}

Normalmente la vena porta hepática (VP) es formada por la confluencia de la vena esplénica y la vena mesentérica superior y se extiende hasta el hilio hepático, donde se divide en las ramas derecha e izquierda, las que a su vez se ramifican progresivamente para irrigar todo el parénquima hepático. Este vaso es responsable de la irrigación funcional del hígado y provee el 70 \% del flujo sanguíneo hepático, a través del cual transporta los productos de la digestión absorbidos desde el tracto gastrointestinal. La irrigación nutricia representa el $30 \%$ restante y procede de la arteria hepática. El drenaje venoso fluye a través de las venas hepáticas, generalmente tres, las que drenan en la porción final de la vena cava inferior, justo antes de atravesar el foramen diafragmático (Moore, 1980). La VP se desarrolla tempranamente, hacia el día 35 de la vida intrauterina a partir de la anastomosis que establecen las venas vitelinas en relación con el duodeno, en especial de la porción infra hepática de la vena vitelina derecha (Larsen, 2003).

La ausencia congénita de la vena porta hepática es una malformación en extremo rara, situación que afecta el desarrollo de la porción infra hepática de la vena vitelina derecha hacia el final del período embrionario. Hasta 2010 se habían reportado sólo 84 casos (Mistinova et al., 2010), sus edades han fluctuado entre recién nacido y 64 años, afectando principalmente a mujeres $(65 \%)$ con un alto índice de morbimortalidad por falla hepática (Mistinova et al.). Un reciente reporte de Hao et al. (2015), eleva esta cifra a 101 ca-

\footnotetext{
Departamento de Anatomía, Escuela de Medicina, Pontificia Universidad Católica de Chile, Santiago, Chile

** Radiología, Escuela de Medicina, Pontificia Universidad Católica de Chile, Santiago, Chile.

**** División de Cirugía, Escuela de Medicina, Pontificia Universidad Católica de Chile, Santiago, Chile.
} 
sos en el mundo desde la primera notificación en 1793. Esta anomalía produce la falta de conexión del hígado con el sistema portal y las venas hepáticas, de modo que el drenaje venoso intestinal es derivado hacia el territorio de las venas sistémicas, generalmente a la vena cava inferior (Ogul et al., 2013).

En 1793 John Abernethy describió por primera vez esta anomalía, detectada en el cadáver de una lactante de 10 meses de edad, más tarde conocida como malformación de Abernethy o derivación porto-sistémica extra-hepática congénita CEPS (Lu et al., 2014). En 1833, Kiernan describió un segundo caso detectado en una niña de 13 años de edad (Alvarez et al., 2002). Desde entonces a la fecha se publicaron otros 99 casos (Mistinova et al.; Hao et al.), incluyendo los últimos tres nuevos casos, dos en niños (Timpanaro et $a l ., 2015)$ y otro en un adolescente (Hao et al.).

Paradojalmente, a pesar de lo impresionante anatómicamente hablando- de esta malformación, su prevalencia no es conocida, en parte porque en algunos pacientes la alteración cursa de manera asintomática y su diagnóstico es tardío o incidental (Emre et al., 2007). Con frecuencia esta afección se asocia a otras alteraciones congénitas, incluyendo anomalías cardíacas, de la vías biliares, metabólicas, diversas derivaciones porto-sistémicas y retardo mental (Mistinova et al.). En la literatura se ha reportado sólo un caso, una niña, donde esta anomalía vascular está asociada a situs inversus abdominis y a la presencia de una vena cava inferior izquierda (Morgan \& Superina, 1994). De los 101 casos reportados a la fecha, un $10,20 \%$ presentó tumores hepáticos benignos y un 30,61\% desarrolló tumores hepáticos malignos (Hao et al.), situación que empeora el pronóstico de esta anomalía (Lu et al.).

En este trabajo presentamos el hallazgo de ausencia de la VP, asociada a una mal rotación intestinal y a la presencia de una vena cava inferior izquierda, en el cadáver de un niño de dos años de edad utilizado con fines docentes en nuestro Departamento de Anatomía. El hallazgo de este tipo de anomalías anatómicas es especialmente útil para nuestros alumnos, ya que resaltan lo formativo de la disección anatómica y las malformaciones que pueden ocurrir en el ser humano en su desarrollo prenatal (Inzunza et al., 1996a; 1996b; Inzunza \& Burdiles 2010a), lo que nos motivó para dar a conocer este caso.

\section{REPORTE DE CASO}

Disección de rutina en un niño de dos años de edad cuya causa de muerte fue una bronconeumonía fulminante.
Se abordó la cavidad peritoneal y se detectó una mal rotación intestinal del tipo "no rotación intestinal" con el yeyuno e íleon ocupando el hemiabdomen derecho y el colon el hemiabdomen izquierdo. Se observó además un meso largo que se extendía desde la unión duodeno-yeyunal hasta la zona correspondiente a la flexura cólica izquierda, donde toda esta porción de intestino aparecía peritonizado, móvil. Sólo el colon descendente se presentó peritonizado fijo y el colon sigmoides exhibía su mesocolon normal.

Al disecar los pedículos vasculares que irrigan al tracto digestivo abdominal, los que fueron mensurados con la ayuda de un pie de metro, se observaron las siguientes características (Fig. 1):

1.- Los vasos mesentéricos inferiores presentaban una disposición normal, la arteria se originaba desde la aorta abdominal y la vena desembocaba en la vena esplénica.

2.- A diferencia de la condición normal, la arteria mesentérica superior se disponía a la derecha de la vena homónima y en la parte inicial de su trayecto daba origen a una artería hepática común aberrante, cuyo calibre alcanzaba 3,57 mm.

3.- La arteria hepática de origen aberrante ascendía a su vez hacia el hilio hepático junto al colédoco, el que se disponía a la derecha de la arteria. Estos dos elementos eran los únicos que transitaban en el borde libre del omento menor.

4.- El tronco celíaco presentaba sólo dos ramas, la arteria esplénica y arteria gástrica izquierda. Desde esta última surgía una rama hepática accesoria (2,52 $\mathrm{mm}$ de calibre) la que a su vez abordaba la zona desnuda del hígado a nivel del borde posterior del órgano, en la zona vecina a la emergencia de las venas hepáticas.

5.- La vena mesentérica superior $(4,67 \mathrm{~mm})$ recibía a la vena esplénica y se curvaba hacia la derecha, pasando detrás del peritoneo parietal posterior para unirse a la vena renal derecha (4,43 mm de calibre). En la convergencia de estas venas desembocaba la vena suprarrenal derecha (Fig. 2).

6.- El confluente venoso "mesentérico-esplénico-renal" así formado, presentaba un trayecto descendente, a la derecha de la aorta abdominal y recibía a la vena gonadal derecha pasando por detrás de la arteria ilíaca común de ese lado, para desembocar en la confluencia de ambas venas ilíacas comunes.

7.- Desde la confluencia venosa señalada, en el punto precedente, la vena cava inferior izquierda (VCII), vaso anómalo de gran calibre (10,29 mm en su inicio) ascendía a la izquierda de la aorta abdominal y en la porción inicial de su trayecto recibía a la vena renal izquierda (4,64 $\mathrm{mm}$ de calibre), vaso 


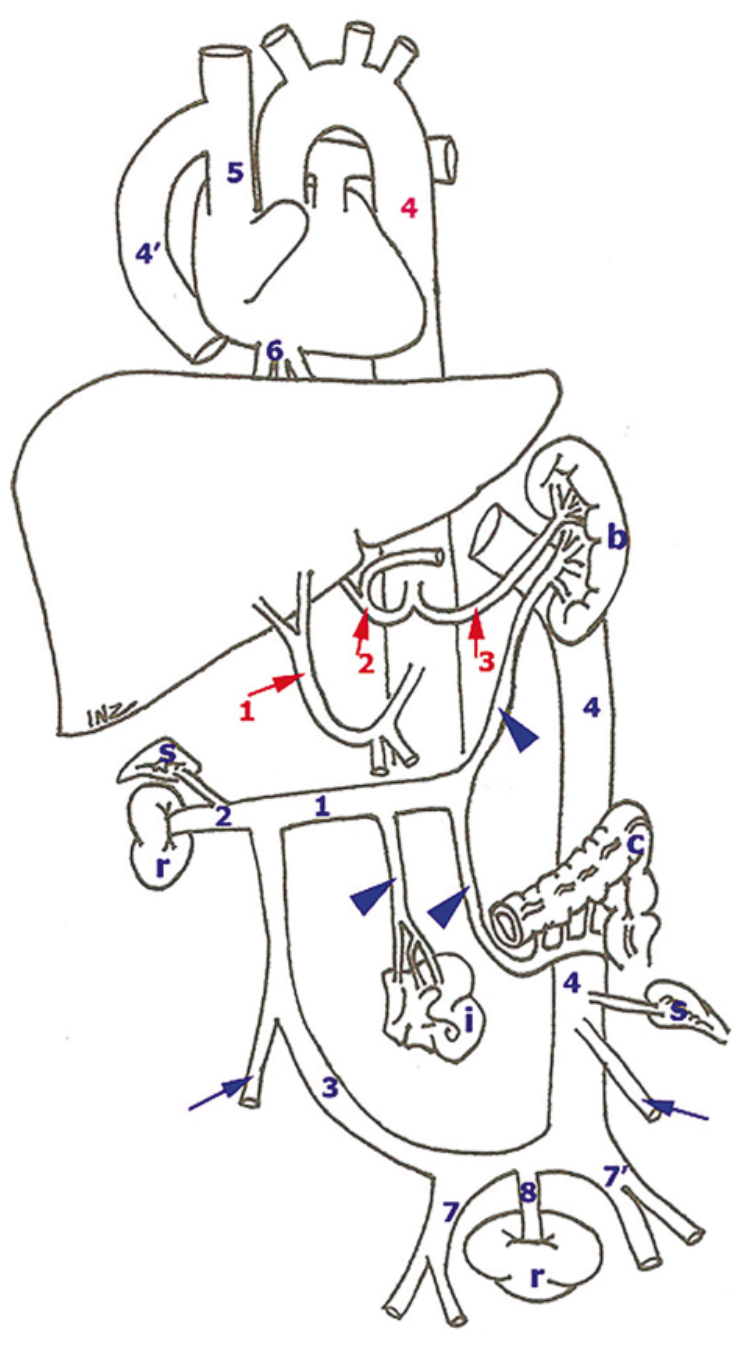

Fig. 1. Esquema que resume los hallazgos del caso presentado: En azul, $1=$ confluente mesentérico-esplénico, formado por las venas esplénica, mesentérica inferior y mesentérica superior (cabezas de flecha azul, de izquierda a derecha respectivamente). $2=$ vena renal derecha, que recibe a la vena adrenal derecha. $3=$ confluente "mesentérico-esplénico-renal", que recibe a la vena gonadal derecha (flecha azul derecha). 4= vena cava inferior izquierda, formada por la confluencia de ambas venas ilíacas comunes ( 7 y 7 '); en esta confluencia drena también la vena renal izquierda (8), originada en el riñón ectópico. La vena cava izquierda recibe separadamente a la vena gonadal (flecha izquierda) y adrenal izquierdas. 4'= porción torácica de la vena cava inferior izquierda que haciendo un cayado desemboca en la vena cava superior (5). $6=$ colector venoso formado por la convergencia de las tres venas hepáticas que desemboca en la zona inferior del atrio derecho. $s=$ glándula suprarrenal. $r=$ riñón. $b=$ bazo. $i=$ intestino delgado. $c=$ colon. En rojo, $1=$ arteria hepática común aberrante, originada desde la arteria mesentérica superior. $2=$ arteria gástrica izquierda, originada desde la aorta abdominal en un tronco común con la arteria esplénica (3); desde 2 surge una rama hepática accesoria, que aborda la zona desnuda del hígado. $4=$ aorta.

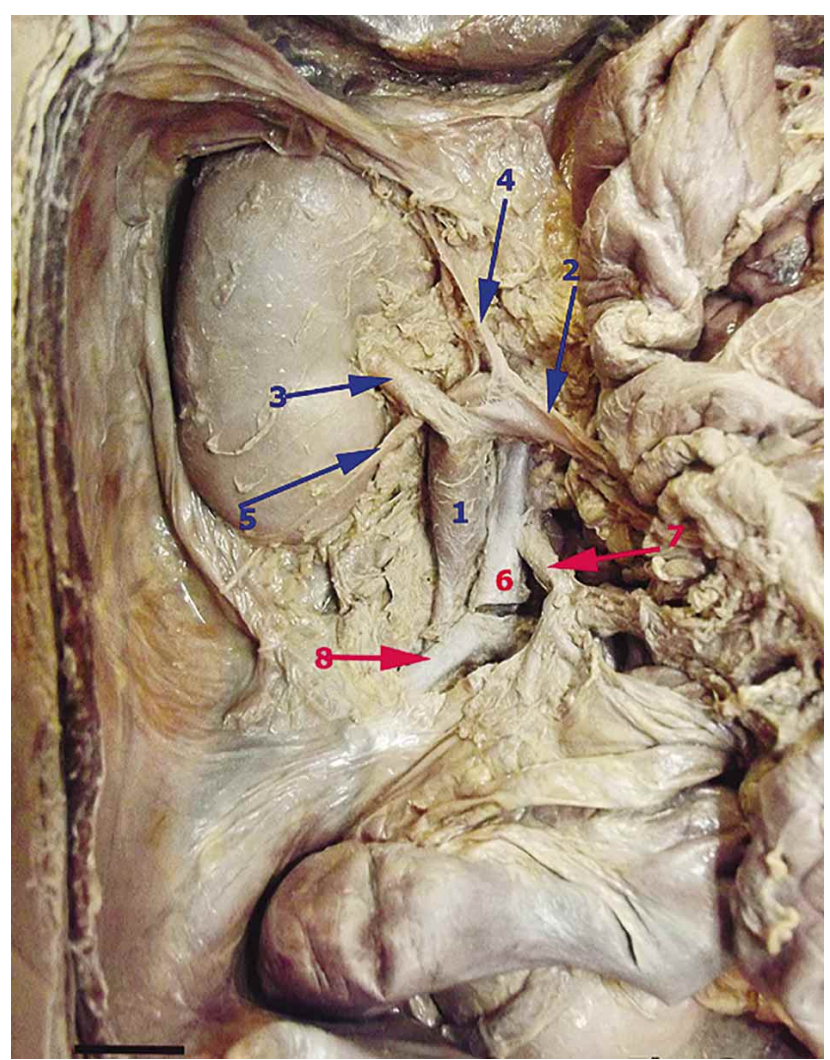

Fig. 2. Disección de la región retroperitoneal derecha que muestra el confluente "mesentérico-esplénico-renal" (1), formado por la unión de la vena mesentérica (2) y la vena renal (3). Las venas adrenal (4) y gonadal (5) de la antimera derecha desembocan en la porción inicial de $1.6=$ porción terminal de la aorta abdominal, $7=$ arteria mesentérica inferior, $8=$ arteria ilíaca común derecha. Barra $=2 \mathrm{~cm}$.

originado en el hilio del riñón de ese lado, el que se encontraba en una situación ectópica, intrapelviana.

8.- Continuando su trayecto ascendente, la VCII recibía las venas gonadal y adrenal de la antimera izquierda, además de las venas lumbares del mismo lado (Fig. 3).

9.- La VCII ascendía luego hacia el tórax, pasando por un arco muscular dispuesto lateral al pilar izquierdo del diafragma. A nivel del mediastino posterior y a la altura del cuerpo vertebral de $\mathrm{T} 9$, la vena se inclinaba hacia la derecha, recibiendo a la vena ácigos accesoria, cruzando por detrás de la aorta descendente y del esófago, adoptando la disposición de la vena ácigos. El vaso luego se curvaba sobre el pedículo pulmonar para desembocar finalmente en la vena cava superior derecha (VCS), la que presentaba un calibre de $13,5 \mathrm{~mm}$.

10.- Las venas hepáticas, en número de tres, se unían para formar un gran colector $(15,4 \mathrm{~mm}$ de calibre) que atravesaba el diafragma para abrirse en la zona inferior del atrio derecho. 


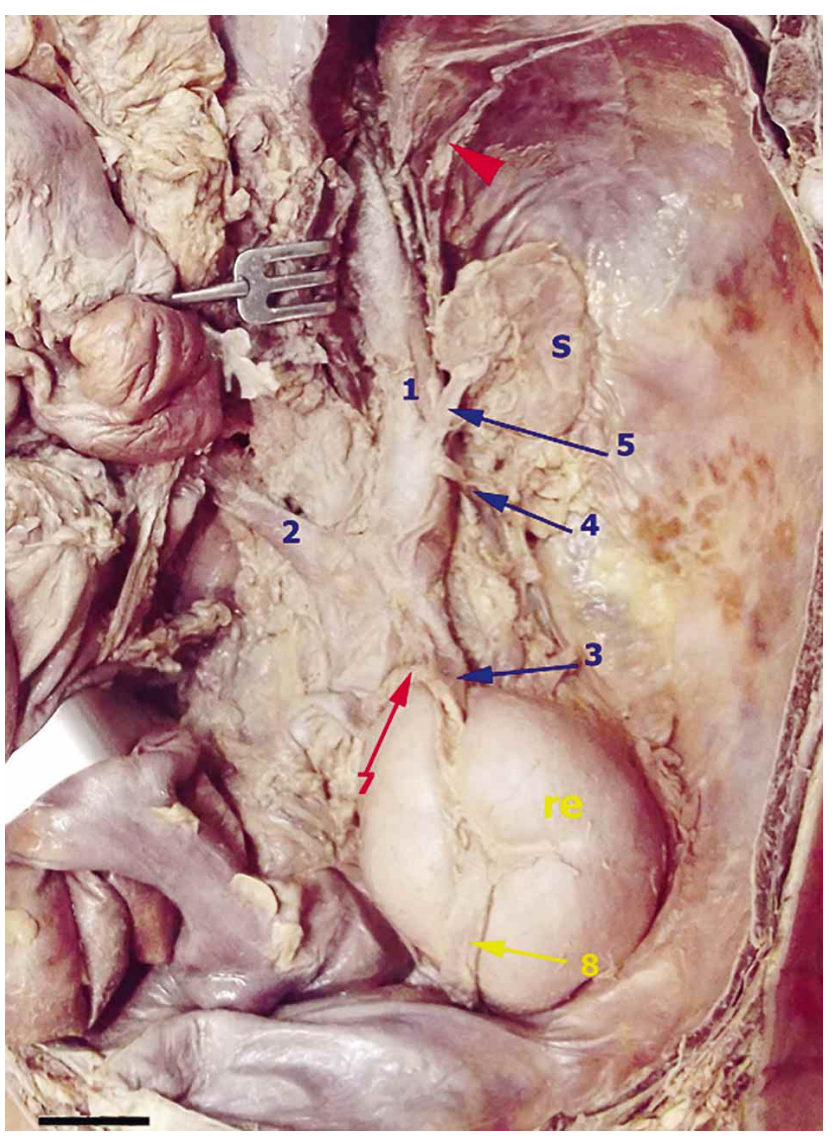

Fig. 3. Disección de la región retroperitoneal izquierda que muestra a la vena cava inferior izquierda (1) que recibe al confluente "mesentérico-esplénico-renal" (2) señalado en la figura 2. El elemento 1 se forma por la unión de: las venas ilíacas comunes, el confluente "mesentérico-esplénico-renal", y la vena renal izquierda (3) que se origina en el riñón ectópico izquierdo (re). $7=$ arteria renal izquierda, $8=$ uréter izquierdo, $\mathrm{d}=$ diafragma, $\mathrm{s}=$ suprarrenal izquierda. Cabeza de flecha $=$ diafragma. La erina está separando el orificio diafragmático por donde cruza 1 hacia el tórax. Barra= $2 \mathrm{~cm}$.

\section{DISCUSIÓN}

Conforme con lo señalado por Morgan \& Superina, las malformaciones porto-sistémicas pueden ser clasificadas de acuerdo a si el parénquima hepático es o no irrigado por las venas mesentéricas. En las malformaciones tipo I, el hígado no es perfundido por la vena porta hepática, la que está ausente, existiendo una derivación total del flujo venoso mesentérico hacia venas sistémicas. En este tipo existirían a su vez dos variedades: las de tipo Ia, donde la vena mesentérica superior y la vena esplénica llegan por separado a la circulación sistémica, y las tipo $\mathrm{Ib}$, donde éstas venas se unen antes de alcanzar el flujo sistémico. Por otro lado, en las malformaciones tipo II, el hígado es parcialmente perfundido por el sistema porta y en esta categoría existen también dos variedades: las de tipo IIa, donde la anomalía es congénita y las tipo IIb, donde la anomalía es adquirida, como puede verse en cuadros con hipertensión portal. Nuestro caso correspondería a una malformación de Abernethy tipo Ib.

El territorio venoso del embrión se encuentra organizado en tres sistemas: i) El sistema vitelino, que drena el tracto gastrointestinal en desarrollo. ii) El sistema umbilical, que transporta sangre oxigenada desde la placenta. iii) El sistema cardinal, que se desarrolla en una secuencia temporal particular, primero las venas cardinales, luego las venas subcardinales y finalmente las venas supracardinales; las venas de este sistema drenan la pared corporal del embrión y las regiones de cabeza y cuello. Hacia el día embrionario 28 estos tres sistemas, que son bilaterales y simétricos, desembocan separadamente en las astas derecha e izquierda del seno venoso (Larsen; Carlson, 2000; Minniti et al., 2002).

Las venas vitelinas establecen anastomosis entre sí y también con colectores venosos del duodeno y del hígado que se desarrollan bajo el septo transverso, siendo estas últimas conexiones las precursoras de los sinusoides hepáticos. Hacia el día embrionario 35 se observa regresión selectiva de la porción caudal de la vena vitelina derecha y de la porción craneal de la vena vitelina izquierda, la que pierde su conexión con el seno venoso. De esta manera, al final del período embrionario, la porción infra hepática de la vena vitelina derecha forma la vena porta, mientras que la porción supra hepática deriva en las venas hepáticas y en el segmento supra hepático de la vena cava inferior.

A su vez, la vena cava inferior (VCI) se forma como producto de la conexión longitudinal de diferentes segmentos del sistema subcardinal, supracardinal y cardinal. Así, la porción inicial de la VCI, formada por el confluente venoso ilíaco (tracto ilíaco), deriva de la anastomosis entre las venas cardinales posteriores. El segmento infrarenal de la VCI (tracto subrrenal), deriva a su vez de la vena supracardinal derecha. El segmento renal (tracto renal), deriva de la anastomosis sub-supracardinal derecha. El segmento de la VCI comprendido entre el hígado y los riñones (tracto hepático), deriva de la vena subcardinal derecha. Por último, el segmento terminal de la VCI deriva de la vena vitelina derecha (Larsen; Moore; Minniti et al.; Inzunza et al., 2011). De los 101 casos reportados a la fecha, sólo uno se presenta asociado a una alteración de la rotación intestinal, situs inversus abdominis, y a la translocación de la vena cava inferior que se dispone a la izquierda (Morgan \& Superina); en nuestro caso la vena cava inferior izquierda está asociada a una mal rotación intestinal. 
De acuerdo con esto, podemos decir que en el caso que aquí presentamos existen anomalías congénitas en los sistemas de las venas vitelinas y de las venas cardinales. De modo tal que la formación del confluente "mesentéricoesplénico-renal" sería secundaria a la involución selectiva de la porción infra hepática de la vena vitelina derecha y al desarrollo de una anastomosis entre este vaso y la vena subcardinal derecha. Esta situación interrumpiría a su vez el desarrollo hacia craneal de VCI, generándose una derivación hacia caudal que involucraría también a la vena supracardinal derecha y a la anastomosis entre las venas cardinales posteriores.

Si a estas anomalías venosas se suma la persistencia del segmento caudal de la vena supracardinal izquierda y su conexión con el extremo caudal de la vena cardinal posterior izquierda, queda diseñado el trayecto de la VCII que observamos en este caso (Fig. 1). De esta manera, el cortocircuito hacia caudal, generado por involución de la porción infra hepática de la vena vitelina derecha, sería derivado hacia la izquierda a través de la VCII. Entonces, la persistencia de la vena supracardinal izquierda y su extensión hacia el tórax permitiría que la sangre venosa de la porción caudal del feto, incluyendo el territorio mesentérico y renal, drene hacia cefálico en la vena cava superior (Larsen; Minniti et al.).

De acuerdo a lo señalado en los puntos 3 y 4 de la descripción del caso, es notable lo que ocurre con la irrigación hepática. Habitualmente, la irrigación nutricia del hígado proviene de la arteria hepática propia en tanto que la funcional se origina desde la vena porta hepática, datos ampliamente tratados en la literatura (Moore et al., 2010; Latarjet \& Ruiz Liard, 2005; Drake et al., 2005; Rouvière \& Delmas, 2005). La anastomosis detectada entre la vena mesentérica superior y la vena renal derecha (confluente "mesentérico-esplénico-renal") modifica el patrón circulatorio clásicamente descrito, enviando la sangre venosa derivada desde el bazo, páncreas e intestinos hacia la VCII, es decir, a la circulación sistémica, sin pasar en primera instancia por el hígado. Esto determina que, en este caso, el hígado sólo reciba irrigación a través de las arterias hepáticas propia y accesoria descritas anteriormente (Fig. 1); vasos que le aportan una irrigación mixta, tanto nutricia como funcional al hígado. Por su parte, el drenaje venoso del hígado fluye hacia el atrio derecho a través de un grueso colector formado por la convergencia de las tres venas hepáticas, vasos que derivan del segmento terminal de la vena vitelina derecha.

La ausencia de la vena porta hepática determina que los ácidos grasos, absorbidos en el intestino y transportados en la forma de quilomicrones, pasen directamente a la circulación sistémica, quedando a disposición de los tejidos periféricos, incluyendo el tejido adiposo y muscular, donde la grasa es acumulada (Nakasaki et al., 1989). En efecto, en el cadáver del niño se observó macrosomía, con un grueso panículo adiposo en los miembros.

Según lo señalado en la literatura, la ausencia de la vena porta se asocia a otras anomalías congénitas. Las anomalías cardíacas como dextrocardia, defectos del septo atrial y/o ventricular y el ducto arterioso permeable (Gadodia $e t$ al., 2011), representarían cambios congénitos adaptativos en respuesta al "efecto congestivo" inducido por el cortocircuito porto-sistémico (Grazioli et al., 2000). En esta línea, al examinar el corazón del niño observamos un defecto del septo interatrial, con un foramen oval permeable y un defecto en el septum primum, este último ubicado en el margen antero inferior de la fosa oval, sobre la válvula tricúspide, tal como lo describe Morison (1913).

En relación con la mal rotación intestinal detectada en el niño, observamos que la unión duodeno-yeyunal se ubicaba a la derecha de la línea media, con las asas proximales de yeyuno en el cuadrante superior derecho del abdomen. Esta disposición, clasificada como una mal rotación intestinal típica (McVay et al., 2007), puede acompañarse con una inversión en la disposición de los vasos mesentéricos superiores, tal como ocurrió en el caso que presentamos.

Considerando los hallazgos de la disección y lo reportado en la literatura, podemos plantear que las malformaciones congénitas que presentaba el niño correspondieron a un hallazgo incidental y no habrían sido la causa directa de su muerte, determinada por una bronconeumonía aguda.

Por ultimo, y no menos importante, este tipo de anomalías anatómicas observadas en cadáveres disecados con fines docentes en nuestro Departamento de Anatomía, tienen un valor formativo indiscutible para nuestros alumnos, quienes pueden comprobar directamente la enorme variabilidad en el desarrollo del ser humano y relacionar su causa y efecto. Además, estas situaciones anómalas permiten aplicar los conocimientos de embriología de nuestros alumnos para explicar su génesis (Inzunza et al., 1996a, 1996b; Inzunza \& Burdiles; Inzunza et al.).

\section{DEDICATORIA}

Dedicado a nuestro coautor el fallecido Dr. Sergio Zúñiga Rocha, un hábil cirujano pediatra, un entretenido narrador de historias médicas, un entrañable amigo y una excelente persona. 
INZUNZA, O.; GARCÍA，C. J.; ZÚÑIGA， S.; INZUNZA, M.; SCHNEEBERGER, D.; SALGADO, G. \& FARFÁN, E. Congenital absence of the hepatic portal vein associated to intestinal malrotation and left-sided inferior vena cava. A case report. Int. J. Morphol., 34(2):665-670, 2016.

SUMMARY: Congenital absence of the hepatic portal vein is an extremely rare malformation that was first described by John Abernethy in 1793. Only 101 cases had been described in the literature until 2015 and most affected females. In this anomaly, also known as congenital extrahepatic porto-systemic shunt (CEPS), intestinal venous drainage is derived towards the territory of the systemic veins and could be associated with other birth defects, including heart and biliary tract anomalies, metabolic diseases, mental retardation. We present the case of a 2-year-old boy who died because of multifocal pneumonia and necropsy showed anatomical findings consistent with this malformation as an incidental finding. The portal vein followed an anomalous course and joined the right renal vein, forming the "mesentericsplenic-renal" venous collector, which then received the right gonadal vein and ended at the confluence of the common iliac veins. In our case this anomaly was associated to intestinal malrotation, aberrant hepatic artery and persistent left inferior vena cava, situation described once in the literature. The finding of these anatomical abnormalities in cadavers has a great teaching value for our undergraduate and graduate students who are learning anatomy and they can also assess the associated clinical.

KEY WORDS: Absence of portal vein; Porto-caval anastomosis; Portal vein development; Intestinal malrotation.

\section{REFERENCIAS BIBLIOGRÁFICAS}

Alvarez, A. E.; Ribeiro, A. F.; Hessel, G.; Baracat, J. \& Ribeiro, J. D. Abernethy malformation: one of the etiologies of hepatopulmonary syndrome. Pediatr. Pulmonol., 34(5):391-4, 2002.

Carlson, B. M. Embriología Humana y Biología del Desarrollo. $2^{\mathrm{a}}$ ed. Madrid, Harcourt, 2000.

Drake, R. L.; Volg, W. \& Mitchell, A. W. M. Gray Anatomía para Estudiantes. Madrid, Elsevier, 2005

Emre, S.; Arnon, R; Cohen, E.; Morotti, R. A.; Vaysman, D. \& Shneider, B. L. Resolution of hepatopulmonary syndrome after auxiliary partial orthotopic liver transplantation in Abernethy malformation. A case report. Liver Transpl., 13(12):1662-8, 2007.

Gadodia, A.; Sharma, R.; Kandpal, H. \& Prashad, R. Congenital absence of portal vein with large inferior mesenteric-caval shunt. Trop. Gastroenterol., 32(3):223-6, 2011.

Grazioli, L.; Alberti, D.; Olivetti, L.; Rigamonti, W.; Codazzi, F.; Matricardi, L.; Fugazzola, C. \& Chiesa, A. Congenital absence of portal vein with nodular regenerative hyperplasia of the liver. Eur. Radiol., 10(5):820-5, 2000.

Hao, Y.; Hong, X. \& Zhao, X. Congenital absence of the portal vein associated with focal nodular hyperplasia of the liver and congenital heart disease (Abernethy malformation): A case report and literature review. Oncol. Lett., 9(2):695-700, 2015.

Inzunza, H. O. \& Burdiles, A. Aberrant left vertebral artery. Int. J. Morphol., 28(1):209-12, 2010.

Inzunza, H. O.; Inzunza, A. M. \& Salgado, A. G. Recurrent retroaortic left renal vein. Report of an uncommon anomaly. Int. J. Morphol., 29(2):33943, 2011.
Inzunza, O.; Prado, M. \& Valenzuela A. Variación del origen de la arteria coronaria derecha. Rev. Chil. Cienc. Med. Biol., 6(1):11-4, 1996 a.

Inzunza, O.; Valenzuela, A.; \& Prado, M. Anastomosis anómala entre las arterias hepática y mesentérica superior. Rev. Chil. Cienc. Med. Biol., 6(2):65$8,1996 \mathrm{~b}$.

Larsen, W. J. Essentials of Human Embriology. Edinburgh, Churchill Livingstone, 1998.

Latarjet, M. \& Ruiz Liard, A. Anatomía Humana. Tomo II . $4^{\mathrm{a}}$ ed. Buenos Aires, Médica Panamericana, 2005.

Lu, J.; Lin, Z.; Liu, H. \& Liu, Z. An unusual presentation of type II Abernethy malformation. Ann. Vasc. Surg., 28(6):1567.e1-4, 2014.

McVay, M. R.; Kokoska, E. R.; Jackson, R. J. \& Smith, S. D. Jack Barney Award. The changing spectrum of intestinal malrotation: diagnosis and management. Am. J. Surg., 194(6):712-7, 2007.

Minniti, S.; Visentini, S. \& Procacci, C. Congenital anomalies of the venae cavae: embryological origin, imaging features and report of three new variants. Eur. Radiol., 12(8):2040-55, 2002.

Mistinova, J.; Valacsai, F. \& Varga, I. Congenital absence of the portal vein-Case report and a review of literature. Clin. Anat., 23(7):750-8, 2010.

Moore, K. L. Clinically Oriented Anatomy. Baltimore, Williams \& Wilkins, 1980.

Moore K. L.; Dalley, A. F. \& Agur, A. M. R. Clinically Oriented Anatomy. 6th ed. Philadelphia, Wolters Kluwer Health/Lippincott Williams \& Wilkins, 2010.

Morgan, G. \& Superina, R. Congenital absence of the portal vein: two cases and a proposed classification system for portasystemic vascular anomalies. J. Pediatr. Surg., 29(9):1239-41, 1994.

Morison, A. Malformation of the Heart-Foramen Primum. Proc. R. Soc. Med., 6(Sect. Study Dis. Child.):21-2, 1913.

Nakasaki, H.; Tanaka, Y.; Ohta, M.; Kanemoto, T.; Mitomi, T.; Iwata, Y. \& Ozawa, A. Congenital absence of the portal vein. Ann. Surg., 210(2):1903,1989 .

Ogul, H.; Bayraktutan, U.; Yalcin, A.; Turan, M. I.; Ozgokce, M.; Eren, S. \& Kantarci, M. Congenital absence of the portal vein in a patient with multiple vascular anomalies. Surg. Radiol. Anat., 35(6):529-34, 2013.

Rouvière, H. \& Delmas, A. Anatomía Humana: Descriptiva, Topográfica y Funcional. 11ª ed. Barcelona, Masson, 2005.

Timpanaro, T.; Passanisi, S.; Sauna, A.; Trombatore, C.; Pennisi, M.; Petrillo, G.; Smilari, P. \& Greco, F. Congenital portosystemic shunt: our experience. Case Rep. Pediatr., 2015:691618, 2015.

\section{Dirección para Correspondencia: \\ Prof. Dr. Oscar Inzunza \\ Departamento de Anatomía Normal \\ Escuela de Medicina \\ Pontificia Universidad Católica de Chile \\ Santiago - CHILE}

Email: oinzunza@med.puc.cl

Recibido : 14-01-2016

Aceptado: 22-03-2016 